\title{
Predictive model for inflammation grades of chronic hepatitis B: Large-scale analysis of clinical parameters and gene expressions
}

\author{
Weichen Zhou ${ }^{1,2,3}$ | Yanyun Ma $^{2}$ | Jun Zhang ${ }^{1}$ | Jingyi Hu ${ }^{1,2}$ | Menghan Zhang ${ }^{2}$ Yi \\ Wang $^{2} \mid$ Yi Li $^{2} \mid$ Lijun Wu ${ }^{1}$ | Yida Pan ${ }^{1} \mid$ Yitong Zhang ${ }^{1,2}$ | Xiaonan Zhang ${ }^{4}$ Xinxin \\ Zhang $^{5}$ | Zhanqing Zhang ${ }^{4}$ | Jiming Zhang ${ }^{6}$ | Hai Li ${ }^{7}$ Lungen Lu $^{8}$ | Li Jin ${ }^{2}$ | Jiucun \\ Wang $^{2}$ | Zhenghong Yuan ${ }^{4,9}$ | Jie Liu ${ }^{1,9}$ \\ ${ }^{1}$ Department of Digestive Diseases of Huashan Hospital, Collaborative Innovation Center for Genetics and Development, Fudan University, Shanghai, China \\ ${ }^{2}$ State Key Laboratory of Genetic Engineering, Collaborative Innovation Center for Genetics and Development, School of Life Sciences and Institutes of Biomedical \\ Sciences, Fudan University, Shanghai, China \\ ${ }^{3}$ Department of Computational Medicine \& Bioinformatics, University of Michigan, Ann Arbor, MI, USA \\ ${ }^{4}$ Shanghai Public Health Clinical Center, Fudan University, Shanghai, China \\ ${ }^{5}$ Department of Infectious Diseases, Ruijin Hospital, Shanghai Jiaotong University School of Medicine, Shanghai, China \\ ${ }^{6}$ Department of Infectious Diseases, Huashan Hospital, Fudan University, Shanghai, China \\ ${ }^{7}$ Department of Gastroenterology, Renji Hospital, Shanghai Jiaotong University School of Medicine, Shanghai, China \\ ${ }^{8}$ Department of Gastroenterology, Shanghai General Hospital, Shanghai Jiaotong University School of Medicine, Shanghai, China \\ ${ }^{9}$ Key Laboratory of Medical Molecular Virology of MOE/MOH, Department of Immunology, Institutes of Biomedical Sciences, Shanghai Medical School, Fudan \\ University, Shanghai, China
}

\section{Correspondence}

Jiucun Wang, PhD, School of Life Sciences,

Fudan University, Shanghai, China.

Email: jcwang@fudan.edu.cn

Jie Liu, MD, PhD, Department of Digestive

Diseases, Huashan Hospital, Fudan University,

Shanghai, China.

Email: jieliu@fudan.edu.cn

and

Zhenghong Yuan, MD, PhD, Shanghai Public

Health Clinical Center, Fudan University,

Shanghai, China.

Email: zhyuan@shaphc.org

\section{Funding information}

This work was supported by grants from the National Natural Science Foundation of China (31521003, 91129702 and 81125001), the Ministry of Science and Technology of China (2006AA02A411), the Major National Science and Technology Program of China (2008ZX10002-002) and the 111 Project (B13016) from Ministry of Education (MOE). Computational support was provided by the High-End Computing Center located at Fudan University.

Handling Editor: Mario Mondelli

\begin{abstract}
Background: Liver biopsy is the gold standard to assess pathological features (eg inflammation grades) for hepatitis $B$ virus-infected patients although it is invasive and traumatic; meanwhile, several gene profiles of chronic hepatitis $B(\mathrm{CHB})$ have been separately described in relatively small hepatitis B virus (HBV)-infected samples. We aimed to analyse correlations among inflammation grades, gene expressions and clinical parameters (serum alanine amino transaminase, aspartate amino transaminase and HBV-DNA) in large-scale CHB samples and to predict inflammation grades by using clinical parameters and/or gene expressions.

Methods: We analysed gene expressions with three clinical parameters in $122 \mathrm{CHB}$ samples by an improved regression model. Principal component analysis and machinelearning methods including Random Forest, K-nearest neighbour and support vector machine were used for analysis and further diagnosis models. Six normal samples were conducted to validate the predictive model.

Results: Significant genes related to clinical parameters were found enriching in the immune system, interferon-stimulated, regulation of cytokine production, antiapoptosis, and etc. A panel of these genes with clinical parameters can effectively
\end{abstract}

Abbreviations: ALT, alanine amino transaminase; AST, aspartate amino transaminase; AUC, area under the ROC curve; CHB, chronic hepatitis B; Cl, confidence interval; DAVID, Database for Annotation, Visualization and Integrated Discovery; HBV, hepatitis B virus; HCC, hepatocellular carcinoma; KNN, K-nearest neighbour; LARS, least angle regression; MDA, mean decrease accuracy; PCA, principal component analysis; RF, random forest; SVM, support vector machine. 
predict binary classifications of inflammation grade (area under the ROC curve [AUC]: $0.88,95 \%$ confidence interval $[\mathrm{Cl}]: 0.77-0.93)$, validated by normal samples. A panel with only clinical parameters was also valuable (AUC: $0.78,95 \% \mathrm{Cl}$ : $0.65-0.86$ ), indicating that liquid biopsy method for detecting the pathology of $\mathrm{CHB}$ is possible.

Conclusions: This is the first study to systematically elucidate the relationships among gene expressions, clinical parameters and pathological inflammation grades in CHB, and to build models predicting inflammation grades by gene expressions and/or clinical parameters as well.

\section{KEYWORDS}

clinical predictive model, gene expressions, $\mathrm{HBV}$ infection, inflammation grades

\section{1 | INTRODUCTION}

In clinic, liver biopsy is a gold standard to directly assess pathological features (eg the inflammation level G) and determine prognosis for hepatitis $B$ virus (HBV)-infected patients. ${ }^{1}$ But it is invasive and traumatic. Serum parameters (eg alanine amino transaminase $[\mathrm{ALT}]$ and aspartate amino transaminase [AST]) are utilized to access the damage of liver and HBV viral infection. ${ }^{1,2}$ In certain cases, these three clinical parameters are necessities for the decision of following appropriate therapy. ${ }^{1,3}$

Microarray is a well-established and widely used technology, which can effectively provide an image of gene expressions. ${ }^{4}$ Researchers have only identified several gene profiles in relative small number of HBV-infected patients, ${ }^{5,6}$ some of which have investigated gene expressions with single clinical parameter, eg ALT or HBV expression. ${ }^{7}$ There are few studies systematically combining clinical parameters, gene expressions and pathological inflammation levels to acquire a comprehensive view of chronic hepatitis $B(\mathrm{CHB})$, not to mention in a large-scale sample size. Other researchers began to explore a liquid biopsy method to assess liver function based on single clinical parameter or in other liver disease (eg chronic hepatitis C). ${ }^{8-10}$ There is barely any effective predictive model for inflammation grades of $\mathrm{CHB}$ right now, and liquid biopsy method is even more elusive.

In this article, we carried out the first study combining three clinical parameters (serum ALT, AST and HBV-DNA), gene microarray data and inflammation grades of $\mathrm{CHB}$. We determined a batch of gene expressions significantly correlated with these clinical continuous parameters and uncovered pathways and networks related to $\mathrm{CHB}$ by comprehensive bioinformatics analyses. More importantly, it is the first time to construct an effective model to diagnose and predict inflammation grades in HBV-infected patients by using these significant gene expressions and/or three clinical parameters, which can help to develop liquid biopsy method for detecting the pathology of $\mathrm{CHB}$.

\section{2 | MATERIALS AND METHODS}

\section{1 | Collection of samples and clinical data}

This study was approved by the ethics committees of Fudan University (Shanghai, China). All subjects provided written informed consents

\section{Key points}

- Correlations among inflammation grades, clinical parameters and gene expressions in chronic hepatitis $\mathrm{B}(\mathrm{CHB})$ patients are only partially enclosed; meanwhile, liquid biopsy prediction of inflammation grades is still unexplored.

- A list of significant genes correlated with clinical parameters was revealed in several functions and pathways from large-scale samples.

- A panel of genes and clinical parameters can effectively predict binary classifications of inflammation grade (area under the ROC curve [AUC]: $0.88,95 \%$ confidence interval [Cl]: 0.77-0.93).

- A panel with only clinical parameters also has a power (AUC: $0.78,95 \% \mathrm{Cl}$ : 0.65-0.86) to predict inflammation, which can be further used in the liquid biopsy method for detecting the pathology of $\mathrm{CHB}$.

according to institutional guidelines. A standardized procedure was established for preservation of liver biopsy sample and RNA extract method. Briefly, after the biopsy was taken, it was quickly submerged in RNAlater, which is an effective stabilizer of tissue RNA, and stored at $4^{\circ}$. The sample was later shipped to a biobank and stored at $-80^{\circ}$ for long-term storage. The workflow of microarray analysis requires rigorous quality control in RNA integrity. Only RNA samples extracted with RIN $>=7.0$ and $28 \mathrm{~S} / 18 \mathrm{~S}>0.7$ were processed further. Four sampling sites must completely follow this standardized procedure, and patients must have same $\mathrm{CHB}$ diagnostic criteria, including HBV persistent infection and $\mathrm{HBsAg}$ positive. Liver biopsy showed varying degrees of inflammatory necrosis, and there is no distribution bias of liver disease grades among these sites. Normal samples were obtained and validated by liver biopsy with non-HBV-infected. Hepatitis samples were obtained by liver biopsy and blood sampling was conducted. The samples with HCV infection or metabolic liver injury (eg fatty liver and chronic alcoholic 
hepatitis) were excluded. After extraction of cRNA, liver tissues were processed by GeneChip Human Genome U133 Plus 2.0 Arrays.

Three clinical parameters were measured in blood. The samples with inexact values $\left(>5 \times 10^{7}\right.$ or $\left.<500\right)$ of HBV-DNA were excluded. The activity of inflammation was measured and confirmed by pathological examination of liver biopsies from two experienced pathologists separately. They were characterized into five grades (G0-4) following the pathological analysis of the biopsies. ${ }^{1,11,12}$

\section{2 | Data processing and bioinformatics analysis}

CEL files were performed by Affymetrix Expression Console. Probe set signals were normalized and summarized by the robust multi-array average algorithm ${ }^{13}$ to adjust different batch effects. All samples passed quality control. The data discussed in this publication have been deposited in NCBI's Gene Expression Omnibus and are accessible through accession number GSE83148.

We normalized the values of parameters, by $\log _{10}$ transformation of HBV-DNA values and min-max normalization of values of ALT and AST. Subsequently, the least angle regression (LARS) algorithm (package $\operatorname{Lars}^{14}$ ) was performed to obtain significant probes that correlated with ALT, AST and HBV-DNA, respectively. We only used the expression data of the samples with valid information. Later, significant probe-level sets were converted to gene-level by using annotation file.

Pathway and gene ontology (GO) enrichment were performed by using the Database for Annotation, Visualization and Integrated Discovery (DAVID, http://david.abcc.ncifcrf.gov/). Cytoscape was applied to build gene networks with geneMANIA plugin. ${ }^{15}$

\section{3 | Principal component analysis and linear regression}

PAST (http://folk.uio.no/ohammer/past) was used to carry out principal component analysis (PCA) and linear regression to investigate expressions of significant genes correlated with ALT and AST. The loading coefficients of significant genes were obtained according to different PCs. The scatter values of HBV-infected samples in each PC were transformed from the expression values of each sample by loading coefficients. In the linear regression (Figure 4D) for PC3, inflammation grades were considered as numerical variables 0-4 and PC3 scatter values were considered as dependent variables, with box plots and fitted lines plotted.

\subsection{Binary classifications of inflammation grades and predictive models by machine- learning methods}

G0 and G1 were considered as mild inflammation and G 2-4 as moderate or severe inflammation. ${ }^{1,12}$ Based on these, binary classifications (mild or exacerbated) of $\mathrm{G}$ were introduced. The expressions of significant genes, the above three clinical parameters and information of sex and age were then utilized to predict these binary classifications of $G$.

Based on the $\mathrm{G}$ classifications, feature selections were conducted by random forest (RF) among significant genes that correlate with either ALT and HBV-DNA, ALT and AST, or AST and HBV-DNA. A gene panel was obtained. In this study, we used K-nearest neighbour (KNN), support vector machine (SVM) and RF to build predictive models for three modules. In general, these are all machine-learning methods for classification and regression. ${ }^{16,17} \mathrm{KNN}$ is a non-parametric algorithm, assigning weights to the contributions of neighbours on the basis of the basic principle of majority voting; SVM is a non-probabilistic binary linear classifier, assigning new examples to one category or the other based on a set of training examples; and RF constructs decision trees by training sets and outputs the class either by the mode of classification or regression of the individual trees. KNN was implemented in MATLAB (Mathworks, Natick, MA, USA). SVM (Package e1071) and RF (Package randomForest) were run by $\mathrm{R}$. Three modules for predictive models were separately built: Module 1 (with information of three clinical parameters and adjustment of sex and age), Module 2 (with genes panel obtained by feature selections) and Module 3 (with all information of selected genes panel, clinical parameters, and adjustment of sex and age). All modules with three predictive methods were performed by five-fold cross-validation to avoid over-fitting. ROC curves were plotted (package ROCR), and the area under the ROC curve (AUC) was calculated (package $p R O C$ ) with 95\% confidence interval (CI). All normal samples were conducted as validations by Module 2 with RF. All packages can be downloaded from Bioconductor (http://www.bioconductor.org).

\section{3 | RESULTS}

\section{1 | Distribution of HBV-infected patients by clinical parameters}

One hundred and twenty-two liver hepatitis tissues infected with HBV were obtained. Of these (Figure 1 and Table S1), 90 had exact quantitative HBV-DNA values (ranging from 603 to $1 \times 10^{9}$ ), and 105 samples had valid quantitative ALT (normal values: 7-40, and abnormal values: 41-1554.3) and AST values (normal values: $10-35$, and abnormal values: 36-706.1). One hundred and nineteen samples were portrayed by $G$ (from G0 to G4), with 34 G0 samples, 33 G1 samples, 31 G2 samples, 15 G3 samples and 6 G4 samples. Six normal samples were all identified as G0.

\subsection{Analysis workflow}

We devised a framework for analysing three clinical parameters, gene microarray data and inflammation grades of $\mathrm{CHB}$ (Figure 2A). After normalization, we manipulated LARS into 90 samples with exact values of HBV-DNA to analyse the significances correlated with HBV-DNA and 105 samples with exact values of ALT or AST to analyse the probes significantly correlated with ALT or AST. After annotation, we finally identified 80 significant genes correlated with serum HBV-DNA, including 48 positive and 32 negative, 96 significant genes (53 positive and 43 negative) correlated with serum ALT, and 92 significant ones ( 45 positive and 47 negative) correlated with serum AST, respectively (Figure $2 \mathrm{~B}$ ). Two genes, IGHA1 and ZNF75A, significantly correlated with both values of serum HBV-DNA and ALT. Sixteen others significantly correlated with both values of serum ALT and AST (Figure 2B and Table S2). 


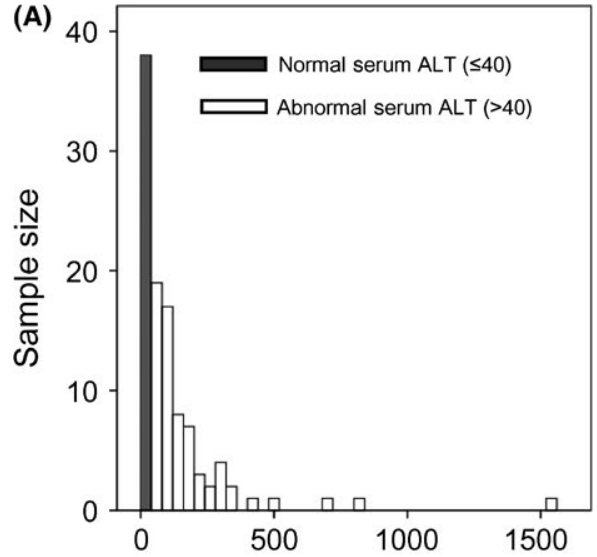

(B)
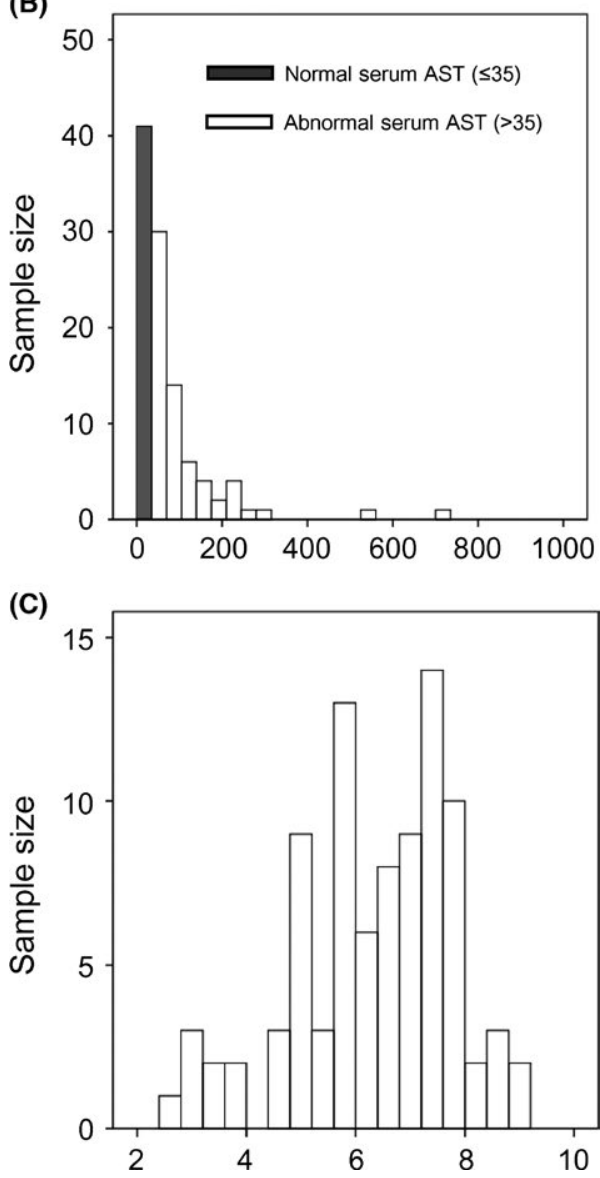

FIGURE 1 Value distribution of three clinical parameters. The $Y$-axis is the number of samples, and the $X$-axis is the value of corresponding serum parameters. $(A)$ and $(B)$ the distributions of serum alanine amino transaminase (ALT) and aspartate amino transaminase (AST) in 105 hepatitis samples, respectively; (C) the distribution of serum hepatitis B virus DNA in 90 hepatitis samples with transformed by $\log _{10}$

\section{3 | Significant gene pathway, GO and gene networks}

A gene pathway consists of a group of interacting components, acting in concert to perform specific biological tasks. ${ }^{18}$ Utilizing the DAVID, we identified seven significant pathways. For HBV-DNA,
hTert transcriptional regulation $\left(P\right.$-value $\left.=2.42 \times 10^{-2}\right)$, lectin-induced complement pathway $\left(P\right.$-value $\left.=4.11 \times 10^{-2}\right)$ and classical complement pathway $\left(P\right.$-value $\left.=4.11 \times 10^{-2}\right)$ were identified. For ALT, B cell activation $\left(P\right.$-value $\left.=2.51 \times 10^{-2}\right)$ was highlighted. For AST, B cell activation pathway was also significantly $\left(P\right.$-value $\left.=4.97 \times 10^{-2}\right)$ enriched, so was pathways in cancer $\left(P\right.$-value $\left.=7.61 \times 10^{-4}\right)$ and pathway of melanoma $\left(P\right.$-value $\left.=4.69 \times 10^{-2}\right)($ Table 1$)$. Significant GO terms are listed in Table S2, which are mostly enriched in immune response (GO: 0006955), apoptosis (GO: 0042981, GO: 0043066, GO: 0060548), positive regulation of cytokine production (GO: 0001819) and etc.

For gene networks, geneMANIA can search large, publicly available biological datasets to illuminate interactions. ${ }^{15}$ Genes were linked by different colour lines, referring to different interactions (Figure 3): co-expression, co-localization, physical interaction and shared protein domains. The number of lines represents the importance of the gene in the network. In the ALT-correlated network, 11 significant genes had more than three lines, eg FLI1, STK17B and ANK2. In the ASTcorrelated network, there are three sub-networks, and DGUOK is an important one which is not in the significant gene list but interacts closely with others. In the HBV-DNA-correlated network, Sept10 and SLC9A3R2 are at the core of two sub-networks.

\section{4 | PCA reveals gene expressions correlated with three biological categories: clinical parameters, gene functions and inflammation grades}

Principal component analysis can be used to determine key variables in gene expression data by using an orthogonal transformation. ${ }^{19}$ By applying PCA to 16 significant gene expressions that correlated with ALT and AST, we obtained three highlighted PCs, each of which can explain more than $10 \%$ of variance: the first (PC1) explained $19.1 \%$ of variance (eigenvalue $=3.052$ ), the second (PC2) explained $13.8 \%$ of variance (eigenvalue $=2.202$ ), and the third (PC3) explained $10.7 \%$ of variance (eigenvalue $=1.705$ ). The more portion of variance it can explain, the more important one component is. We thereby were figuring out biological meanings behind these corresponding components.

In Figure 4A, genes with positive loading coefficients in PC1 are the same ones that positively correlate with serum ALT and AST, and the others with negative loading coefficients have negative correlations. Therefore, PC1 mainly represents the correlative effects of serum ALT and AST.

According to loading coefficients in PC2 (Figure 4B), DLX3, PRDX2 and $Y B X 1$ are enriched in the GO term regarding regulation of transcription with positive coefficients (Table S2). TTLL4, TTLL7 and DCTN4 are enriched in microtubules, and IGF1R and NRXN1 are related to axon-genesis, all of which represent significant genes with negative coefficients correlated with the function of cell cytoskeleton. Therefore, PC2 mainly represents the functional differentiation of genes as serum ALT and AST levels are changing in the HBV-infected patients.

For PC3 (Figure 4C), we carried out a linear regression analysis between inflammation grades of $\mathrm{CHB}$ and scatter values of each sample generated by loading coefficients in PC3 and found a significant 
(A)

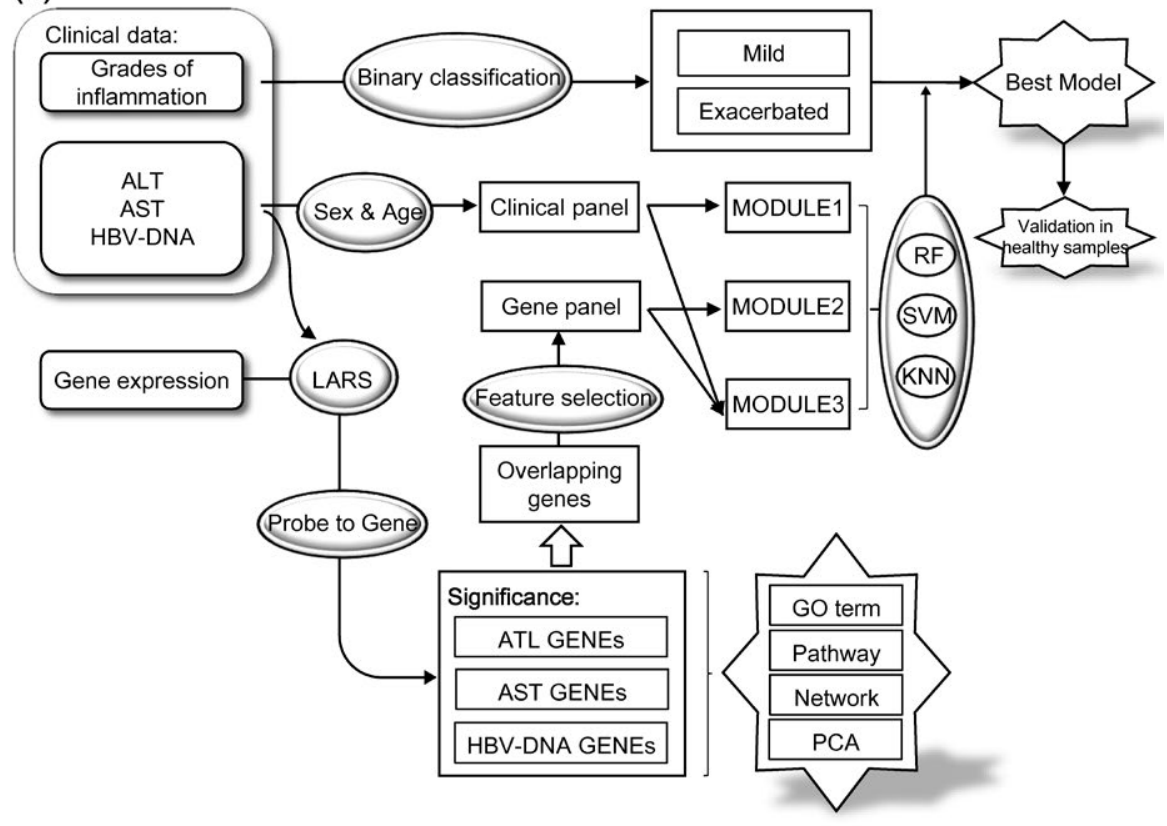

(B)

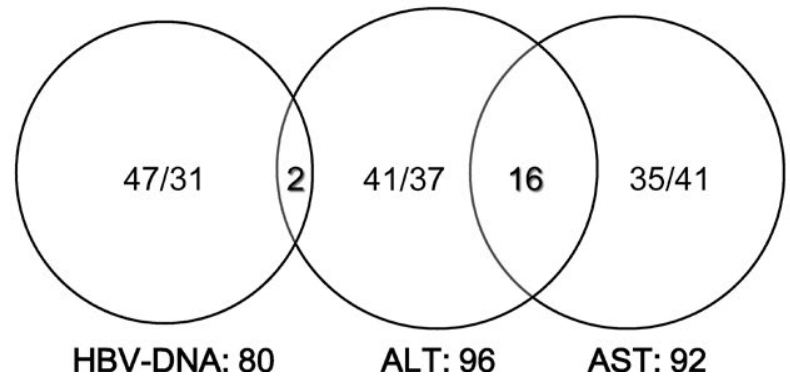

FIGURE 2 Workflow for this study and Venn diagram of significant genes for three clinical parameters. (A) Workflow for analysing three clinical parameters, gene microarray data and inflammation grades of chronic hepatitis B; (B) the Venn diagram of significant genes, including left circle representing significant genes (positive vs negative) correlated with hepatitis $B$ virus (HBV)-DNA, the middle representing significant genes correlated with alanine amino transaminase (ALT), and the right representing significant genes correlated with aspartate amino transaminase (AST). The intersection sets are significant genes shared in the results of HBV-DNA and ALT and AST, respectively

TABLE 1 Significant pathways correlated with three clinical parameters $(P$-values $<.05)$

\begin{tabular}{lllll} 
Type & Database & P-value & Genes & Term \\
\hline ALT & BBID & .0251 & IGHG3, POU2F2, IGHM & B cell activation \\
AST & KEGG & .0008 & IGF1R, FGF16, SMAD3, MDM2, BRCA2, BIRC5, ITGB1, TRAF4 & Pathways in cancer (hsa05200) \\
& KEGG & .0469 & IGF1R, FGF16, MDM2 & Melanoma (hsa05218) \\
& BBID & .05 & IGHG1, POU2F2 & B cell activation \\
\hline HBV-DNA & BIOCARTA & .0241 & SP1, WT1 & Overview of telomerase protein component \\
& & & & gene hTert transcriptional regulation \\
& BIOCARTA & .0411 & C4A, C4B & Lectin-induced complement pathway \\
& BIOCARTA & .0411 & C4A, C4B & Classical complement pathway
\end{tabular}

Three databases (BBID, KEGG and BIOCARTA) were subjected to pathway enrichment analysis. ALT, alanine amino transaminase; AST, aspartate amino transaminase; $\mathrm{HBV}$, hepatitis $\mathrm{B}$ virus.

linear correlation between them $\left(P\right.$-value $=6.69 \times 10^{-3}$; Figure 4D). Therefore, PC3 mainly explains a linear correlation between inflammation grades and gene expressions.

\subsection{Random forest model efficiently diagnoses the inflammation grades in $\mathrm{CHB}$}

According to PCA, there is a correlation between inflammation grades of $\mathrm{CHB}$ and gene expression in PC3. Inspired from this, we further constructed diagnosis models to predict inflammation grades based on the 18 sharing significant genes (two correlated with ALT and HBV-DNA and 16 correlated with ALT and AST).

A binary classification of $G$ was introduced based on the categories of all inflammation grades. ${ }^{1,3}$ By utilizing RF, a gene panel with nine genes (DLX3, ALPK1, YBX1, ZNF75A, SPP2, TTLL4, TTLL7, AGAP3 and $D C T N 4)$ among 18 significant ones for binary classification of $G$ were selected. RF, SVM and KNN were further used to construct predictive models, with the involvement of three clinical phenotypic parameters and adjustment of sex and age. To remove the impact of missing data on results, we only utilized 81 samples with valid information of 


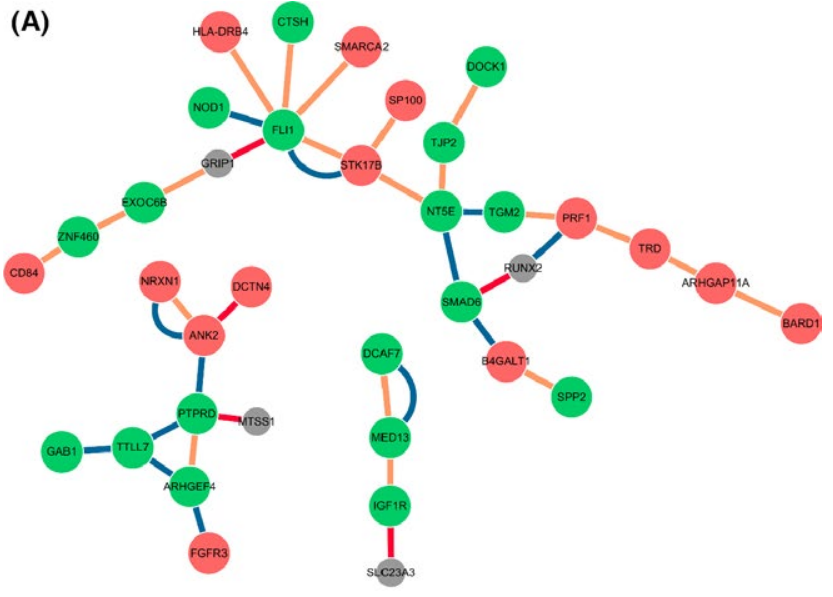

(B)

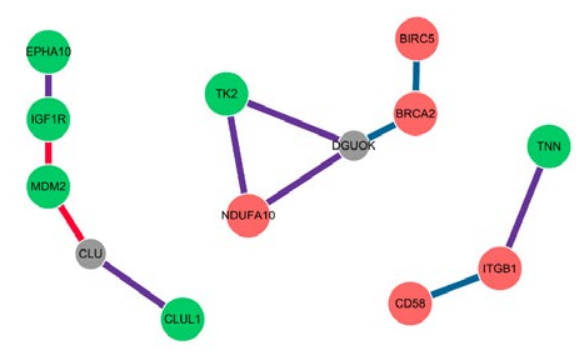

(C)

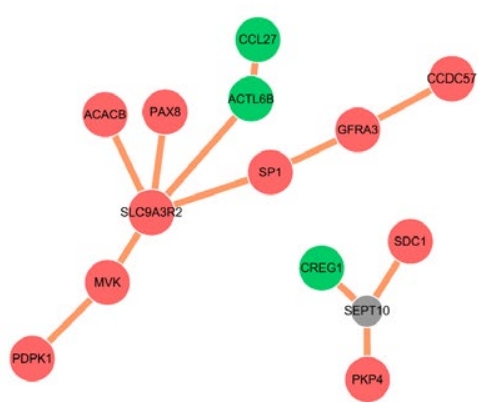

FIGURE 3 Networks generated by significant genes correlated with three clinical parameters. (A) Networks correlated with alanine amino transaminase; (B) and (C) networks correlated with aspartate amino transaminase and hepatitis $B$ virus-DNA, respectively. The red circles represent positively correlated genes and the green represents negative ones. The grey circles represent important genes which are not in the significant gene lists but interact closely with significances. The lines interlinking two genes represent the type of interaction between two genes: orange lines represent co-expression, dark blue ones represent co-localization, red ones represent physical interaction and purple ones represent protein domain sharing

HBV-DNA, ALT, AST, sex and age (Table S1). Sensitivity, specificity and classification accuracy of each method are shown in Table 2, and ROC curves are plotted in Figure 5. All values are averaged in five-fold validations and values of AUC are shown with $95 \% \mathrm{Cl}$, according to different predictive modules and models.

Using genes panel, Module 2 generally performed better than Module 1 by using clinical parameters, based on the results of SVM (0.749 vs 0.734$)$, KNN (0.723 vs 0.729 ) and RF (0.801 vs 0.784 ; Table 2). Notably, when combining all information (Module 3), the predictive power of $\mathrm{KNN}(0.806,95 \% \mathrm{Cl}$ : 0.711-0.898) and RF $(0.880$,
95\% Cl: 0.771-0.933) increased dramatically. More importantly, even though the powers of Module 1 are relatively low (RF: 0.784 , SVM: 0.734 and KNN: 0.729), it is still an improvable model by only using clinical parameters to predict inflammation grades, indicating that liquid biopsy method for detecting the pathology of $\mathrm{CHB}$ is possible. Lastly, we carried out validations by conducting Module 2 of RF method on six normal samples. All six samples were predicted as mild inflammation (G0 or G1), with predicting probability up to $0.827 \pm 0.037$. In conclusion, RF is the most powerful model for the diagnosis of inflammation grades of $\mathrm{CHB}$ when combining expressions of nine genes, three clinical parameters, sex and age.

\section{4 | DISCUSSION}

In this study, we considered clinical parameters as continuous variables and analysed gene expressions by an advanced regression algorithm (LARS), which is more efficient to obtain significant genes than regular linear regression method. ${ }^{14}$ Besides, in $\mathrm{CHB}$ samples, part of them have a normal level of ALT, AST or inflammation grade (G0), which can be considered as baseline values in LARS analysis, PCA and predictive models, as healthy controls in the regular case-control study. Moreover, to maximize the utilization of all information and eliminate the impact of missing data, we discarded samples with missing data in separated steps.

Several genes and pathways correlated with HBV infection and immune response were discovered. TRD, CD84, HLA-DRB4 and B cell activation pathway (Table 1) with genes IGHG3, POU2F2 and IGHM positively correlated with ALT values, suggesting that a proliferation of immune cells and regeneration of liver cells occurs as an increase of serum ALT after HBV infection. Intriguingly, though the gene expression profiles came from a mix of different types of cells, these inflammationrelated genes and pathway indicate the inflammatory cells mixing with hepatic cells may have contributed to the overall gene expression patterns as HBV infection getting worse. In the core of ALT-correlated network (Figure 3A), STK17B with positive correlation was reported to form a novel signalling module which controls calcium homeostasis following T cell activation. ${ }^{20}$ Another core gene PRF1 was reported as an important role in liver cell injury after HBV infection ${ }^{21}$ and HBV-DNA cleanup. ${ }^{22}$ Additionally, in the HBV-DNA network (Figure $3 C$ ), a core significant gene SLC9A3R2, co-expressing with $A C A C B$ and SP1, is a membrane transporter of HBV and HDV entry. ${ }^{23}$ The AST positive specific-related gene CD58 (Figure 3B) was also found related to the microtubule and immune response system and significantly increased with the severity of HBV infection. ${ }^{24}$ Intriguingly, by utilizing interferon-stimulated genes datasets (Interferome: http://interferome.its.monash.edu.au), we found seven interferon-stimulated genes that are significantly correlated with serum HBV-DNA. MKX, TSNARE1 and EFR3A are positively and ACSF3, H2AFJ, XRN1 and ZNF677 are negatively correlated with the increasing value of serum HBV-DNA in infected hepatocytes.

In the AST-related pathway, pathways related to cancer were found (Table 1), supported by the fact that an increasing serum AST often indicates a severe progression of liver cell damage. SP1 and WT1, clustered 

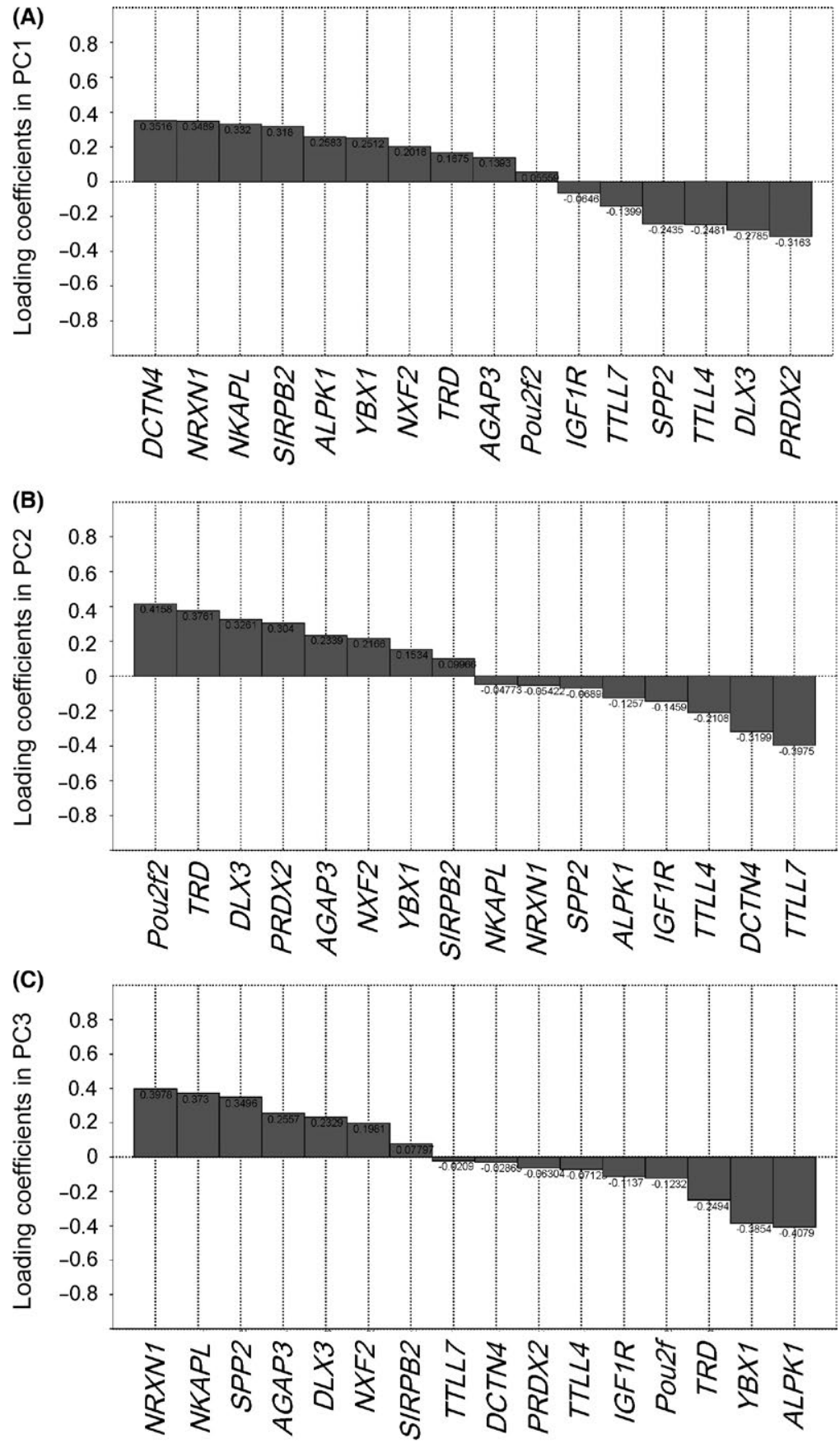

(D)

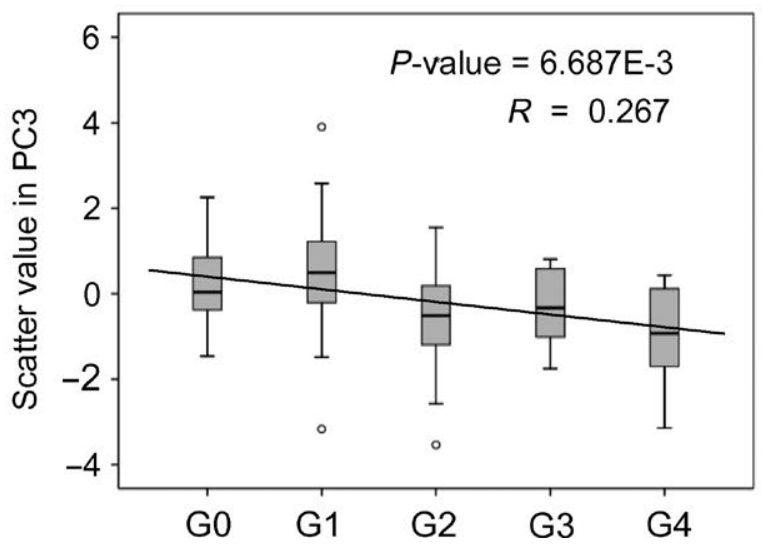

FIGURE 4 Principal component analysis of 16 significant genes correlated both with alanine amino transaminase and aspartate amino transaminase. (A) The plot of genes for PC1 with 10 positive and 6 negative loading coefficients; (B) the genes for PC2 with 8 positive and 8 negative coefficients; (C) the genes for PC3 with 7 positive and 9 negative coefficients; (D) boxplot between G and PC3 scatter values of 102 hepatitis $B$ virus-infected samples. Fitted linear regression lines, $R$ value and $P$-values are shown 
TABLE 2 Specificity, sensitivity, accuracy of classification and area under the ROC curve (AUC) of predictive modules based on three methods with five-fold cross-validation
FIGURE 5 ROC curves based on three predictive models and three modules with five-fold cross-validations. Mean boxplot curves for each model are shown with the values of area under the ROC curve (AUC) and $95 \%$ confidence interval, according to different predictive modules (using three clinical parameters only, nine genes only, or clinical parameters and genes). Dotted curves represent five-fold validations of each experiment

\begin{tabular}{llllll} 
& Specificity & Sensitivity & Accuracy & AUC $(95 \% \mathrm{Cl})$ & Module \\
\hline SVM & 0.6053 & 0.7209 & 0.6667 & $0.7339(0.5832-0.8146)$ & Module 1 \\
& 0.6579 & 0.6744 & 0.6667 & $0.7489(0.5832-0.8165)$ & Module 2 \\
& 0.6579 & 0.6512 & 0.6543 & $0.7093(0.5849-0.8129)$ & Module 3 \\
\hline \multirow{2}{*}{ KNN } & 0.5802 & 0.7971 & 0.6931 & $0.7286(0.6244-0.8407)$ & Module 1 \\
& 0.6938 & 0.8150 & 0.7187 & $0.7226(0.6543-0.8604)$ & Module 2 \\
& 0.6178 & 0.8857 & 0.7666 & $0.8057(0.7108-0.8982)$ & Module 3 \\
\hline \multirow{2}{*}{ RF } & 0.6053 & 0.7674 & 0.6914 & $0.7841(0.6450-0.8562)$ & Module 1 \\
& 0.6842 & 0.7209 & 0.7037 & $0.8015(0.6903-0.8874)$ & Module 2 \\
& 0.6842 & 0.7674 & 0.7284 & $0.8800(0.7710-0.9328)$ & Module 3
\end{tabular}
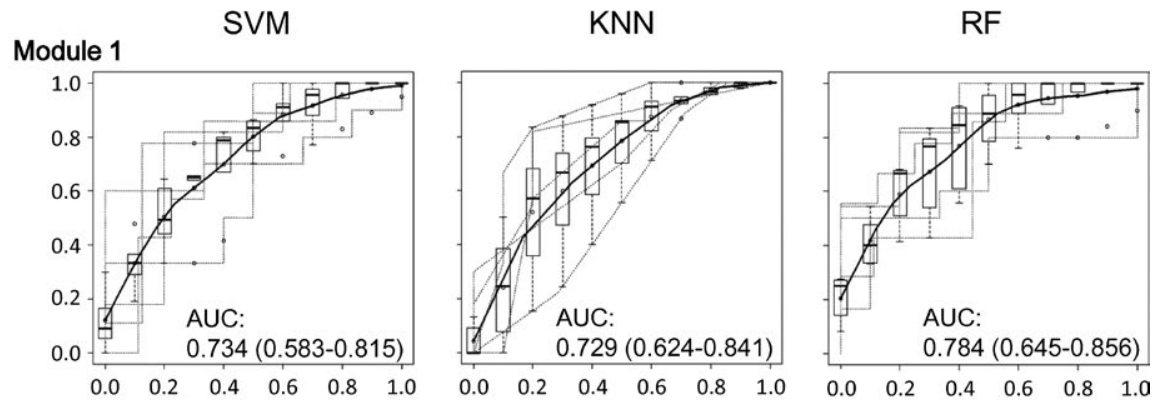

Module 2
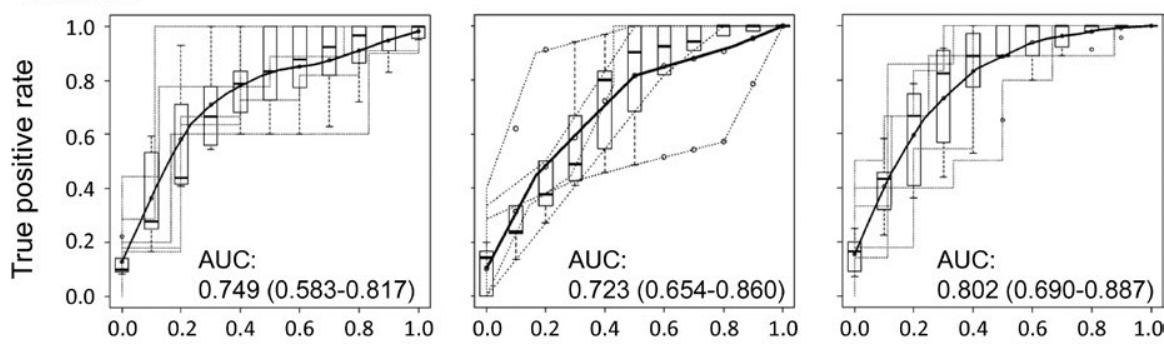

\section{Module 3}
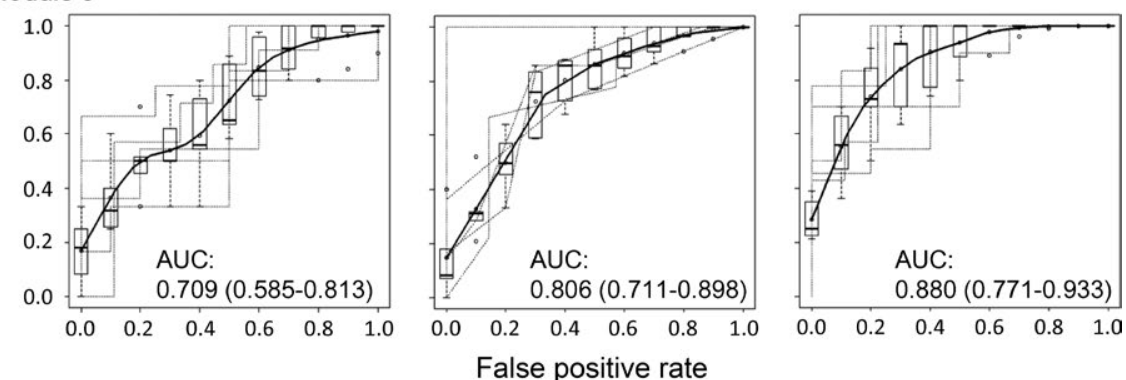

in the pathway of $h$ Tert transcriptional regulation (Table 1), are reported to significantly correlate with $\mathrm{HBx}$ expression ${ }^{25}$ and hepatocellular carcinoma (HCC) development. ${ }^{26-28}$ Interestingly, among 80 significant genes correlated with HBV-DNA, 8 (10\%) of them have been reported to correlate with $\mathrm{HCC}^{26-30}$ or other cancers, ${ }^{22,31,32}$ indicating that they may also play important roles in the progression from HBV-induced inflammation to HCC. Gene IGHA1, which shares significant positive correlation with HBV-DNA and ALT, is also reported involving in gastric tumorigenesis. ${ }^{33}$

Notably, in PCA of expression data of 16 significant genes, five principal components (PCs) had an eigenvalue more than 1 and explained $58.7 \%$ of variance in total. The top three PCs can reveal specific biological insights and explain $43.5 \%$ of variance. In the feature selection of predictive model, nine genes were selected based on their mean decrease accuracy (MDA): YBX1 (MDA=47.8), ALPK1 (MDA=28.0), ZNF75A (MDA=18.2), SPP2 (MDA=13.2), DCTN4 (MDA=12.3), AGAP3 (MDA=7.95), DLX3 (MDA=6.86), TTLL4 (MDA=4.68) and TTLL7 $(M D A=2.57)$. All genes above were mainly related to protein phosphorylation, transcription functions and the major histocompatibility complex. Five of them are related to transcription, indicating the importance between transcription and inflammation grades.

For predictive models, three modules were conducted separately to find the most appropriate model. We suggest RF as a machinelearning black box to aid in prediction and diagnosis for binary classification of inflammation grade of $\mathrm{CHB}$, which has an effective power 
(0.880) with the help of three indispensable clinical parameters and nine genes. In previous studies, there established a predictive model by $\mathrm{Xu}$ et al. ${ }^{9}$ using red blood cell distribution width value, ALT and other blood parameters (albumin and platelet) from 446 patients to predict CHB inflammation with highest AUC of 0.765. However, we have had a relative higher power of AUC of 0.784 (RF, AUC: 0.784 , $95 \% \mathrm{Cl}: 0.65-0.86)$ by using the three clinical parameters from 81 samples to predict inflammation grades in the present study. More samples and studies are highly required based on our models, which may substitute liver biopsy by liquid biopsy method into a practical clinic protocol to characterize the pathological inflammation.

In conclusion, we carried out the first analysis of large-scale HBVinfected samples by combining gene expressions data and three clinical parameters (ALT, AST and HBV-DNA). We considered the parameters as continuous variables and found differentially expressed genes related to these parameters. Most of these significant genes are enriched in immune response, interferon-stimulated, anti-apoptosis and cell proliferation. Some important ones are also reported to correlate with HCC or other cancers.

We found that genes correlated with clinical parameters provide insights for inflammation grades of CHB. We thereby constructed models with novel panels and validated by six normal samples, which can effectively predict binary classifications of inflammation and aid in the diagnosis of CHB. Notably, the novel panel with only clinical parameters was quite valuable, indicating that liquid biopsy method for detecting the pathology of $\mathrm{CHB}$ is possible.

\section{ACKNOWLEDGEMENTS}

We thank Weilin Pu, Kelin Xu, Hua Dong, Chao Chen, Qianqian Peng, Feng Qian and Catherine Ketcham for their critical suggestions.

\section{CONFLICT OF INTEREST}

The authors do not have any disclosures to report.

\section{REFERENCES}

1. Chinese Society of Hepatology CMA, Chinese Society of Infectious Diseases CMA,Hou JL, et al. The guideline of prevention and treatment for chronic hepatitis B: a 2015 update. Zhonghua Gan Zang Bing Za Zhi. 2015;23:888-905.

2. Li W, Zhao J, Zou Z, et al. Analysis of hepatitis B virus intrahepatic covalently closed circular DNA and serum viral markers in treatment-naive patients with acute and chronic HBV infection. PLoS One. 2014;9:e89046.

3. ter Borg MJ, van Zonneveld M, Zeuzem S, et al. Patterns of viral decline during PEG-interferon alpha-2b therapy in HBeAg-positive chronic hepatitis $\mathrm{B}$ : relation to treatment response. Hepatology. 2006;44:721-727.

4. Barrett T, Edgar R. Mining microarray data at NCBI's Gene Expression Omnibus (GEO)*. Methods Mol Biol. 2006;338:175-190.

5. He D, Liu ZP, Honda M, et al. Coexpression network analysis in chronic hepatitis $B$ and $C$ hepatic lesions reveals distinct patterns of disease progression to hepatocellular carcinoma. J Mol Cell Biol. 2012;4:140-152.
6. Ura S, Honda M, Yamashita T, et al. Differential microRNA expression between hepatitis $B$ and hepatitis $C$ leading disease progression to hepatocellular carcinoma. Hepatology. 2009;49:1098-1112.

7. He D, Li M, Guo S, et al. Expression pattern of serum cytokines in hepatitis B virus infected patients with persistently normal alanine aminotransferase levels. J Clin Immunol. 2013;33:1240-1249.

8. Castera L. Noninvasive methods to assess liver disease in patients with hepatitis B or C. Gastroenterology. 2012;142:1293-1302 e4.

9. Xu WS, Qiu XM, Ou QS, et al. Red blood cell distribution width levels correlate with liver fibrosis and inflammation: a noninvasive serum marker panel to predict the severity of fibrosis and inflammation in patients with hepatitis B. Medicine (Baltimore). 2015;94:e612.

10. Praneenararat S, Chamroonkul N, Sripongpun P, et al. HBV DNA level could predict significant liver fibrosis in $\mathrm{HBeAg}$ negative chronic hepatitis B patients with biopsy indication. BMC Gastroenterol. 2014;14:218.

11. Desmet VJ, Gerber M, Hoofnagle JH, et al. Classification of chronic hepatitis: diagnosis, grading and staging. Hepatology. 1994;19:1513-1520.

12. Bedossa P, Poynard T. An algorithm for the grading of activity in chronic hepatitis C. The METAVIR Cooperative Study Group. Hepatology. 1996;24:289-293.

13. Irizarry RA, Hobbs B, Collin F, et al. Exploration, normalization, and summaries of high density oligonucleotide array probe level data. Biostatistics. 2003;4:249-264.

14. Efron B, Hastie T, Johnstone I, et al. Least angle regression. Ann Stat. 2004;32:407-499.

15. Montojo J, Zuberi K, Rodriguez H, et al. GeneMANIA Cytoscape plugin: fast gene function predictions on the desktop. Bioinformatics. 2010;26:2927-2928.

16. Díaz-Uriarte R, Alvarez de Andrés S. Gene selection and classification of microarray data using random forest. BMC Bioinformatics. 2006;7:3.

17. Wang Y, Li Y, Pu W, et al. Random bits forest: a strong classifier/regressor for big data. Sci Rep. 2016;6:30086.

18. Peng G, Luo L, Siu H, et al. Gene and pathway-based second-wave analysis of genome-wide association studies. Eur J Hum Genet. 2010;18:111-117.

19. Raychaudhuri S, Stuart JM, Altman RB. Principal components analysis to summarize microarray experiments: application to sporulation time series. Pac Symp Biocomput. 2000;455-466.

20. Newton RH, Leverrier S, Srikanth S, et al. Protein kinase D orchestrates the activation of DRAK2 in response to TCR-induced $\mathrm{Ca} 2+$ influx and mitochondrial reactive oxygen generation. J Immunol. 2011;186:940-950.

21. Lee JY, Chae DW, Kim SM, et al. Expression of FasL and perforin/ granzyme B mRNA in chronic hepatitis B virus infection. J Viral Hepat. 2004;11:130-135.

22. Hofmann I, Schlechter T, Kuhn C, et al. Protein p0071-an armadillo plaque protein that characterizes a specific subtype of adherens junctions. J Cell Sci. 2009;122:21-24.

23. Ni Y, Lempp FA, Mehrle S, et al. Hepatitis B and D viruses exploit sodium taurocholate co-transporting polypeptide for species-specific entry into hepatocytes. Gastroenterology. 2014;146:1070-1083.

24. Li J, Qi B, Chen P, et al. The expression of CD2 in chronic HBV infection. Cell Mol Immunol. 2008;5:69-73.

25. Park IY, Sohn BH, Yu E, et al. Aberrant epigenetic modifications in hepatocarcinogenesis induced by hepatitis $B$ virus $X$ protein. Gastroenterology. 2007;132:1476-1494.

26. Kou XX, Hao T, Meng Z, et al. Acetylated Sp1 inhibits PTEN expression through binding to PTEN core promoter and recruitment of HDAC1 and promotes cancer cell migration and invasion. Carcinogenesis. 2013;34:58-67.

27. Horikawa I, Barrett JC. Transcriptional regulation of the telomerase hTERT gene as a target for cellular and viral oncogenic mechanisms. Carcinogenesis. 2003;24:1167-1176. 
28. Uesugi $\mathrm{K}$, Hiasa $\mathrm{Y}$, Tokumoto $\mathrm{Y}$, et al. Wilms' tumor 1 gene modulates Fas-related death signals and anti-apoptotic functions in hepatocellular carcinoma. J Gastroenterol. 2013;48:1069-1080.

29. Li HG, Xie DR, Shen XM, et al. Clinicopathological significance of expression of paxillin, syndecan-1 and EMMPRIN in hepatocellular carcinoma. World J Gastroenterol. 2005;11:1445-1451.

30. Song Z, Li R, You N, et al. Loss of heterozygosity of the tumor suppressor gene $\mathrm{Tg} 737$ in the side population cells of hepatocellular carcinomas is associated with poor prognosis. Mol Biol Rep. 2010;37:4091-4101.

31. Raimondi C, Chikh A, Wheeler AP, et al. A novel regulatory mechanism links PLCgamma1 to PDK1. J Cell Sci. 2012;125:3153-3163.

32. Abuli A, Fernandez-Rozadilla C, Giraldez MD, et al. A two-phase case-control study for colorectal cancer genetic susceptibility: candidate genes from chromosomal regions 9q22 and 3q22. Br J Cancer. 2011;105:870-875.
33. Rajkumar T, Vijayalakshmi N, Gopal G, et al. Identification and validation of genes involved in gastric tumorigenesis. Cancer Cell Int. 2010;10:45.

\section{SUPPORTING INFORMATION}

Additional Supporting Information may be found online in the supporting information tab for this article.

How to cite this article: Zhou W, Ma Y, Zhang J, et al. Predictive model for inflammation grades of chronic hepatitis B: Largescale analysis of clinical parameters and gene expressions. Liver Int. 2017;37:1632-1641. https://doi.org/10.1111/liv.13427 